\title{
Ezetimibe, cardiovascular risk and atherogenic dyslipidaemia
}

Manfredi Rizzo, Giovam Battista Rini

Department of Internal Medicine and Medical Specialties, University of Palermo, Italy

Submitted: 24 February 2010

Accepted: 25 February 2010

Arch Med Sci 2011; 7, 1: 5-7

DOI: 10.5114/aoms.2011.20597

Copyright $\odot 2011$ Termedia \& Banach

\begin{abstract}
Ezetimibe is a selective cholesterol absorption inhibitor with an excellent sideeffect profile, able to reduce low-density lipoprotein (LDL) cholesterol by $15-25 \%$ from baseline in monotherapy and on top of statins and fibrates. Yet, it seems that ezetimibe produces quantitative rather than qualitative changes in LDL, with small net effects on atherogenic dyslipidaemia. This is supported by findings from the Ezetimibe and Simvastatin in Hypercholesterolemia Enhances Atherosclerosis Regression (ENHANCE) study on atherosclerosis progression, where the addition of ezetimibe to simvastatin in patients with heterozygous familial hypercholesterolaemia did not affect the mean change in carotid intimamedia thickness, although a significant reduction in LDL cholesterol levels was observed. The Simvastatin and Ezetimibe in Aortic Stenosis (SEAS) study has further shown that combination treatment with simvastatin significantly reduced LDL cholesterol levels in patients with aortic stenosis, but did not affect the primary end point of aortic valve and cardiovascular events, although a significant reduction in the risk of ischaemic events was reported. Formal cardiovascular outcome trials are underway and these will provide additional insights into the long-term effects of ezetimibe on clinical events as well as on atherogenic dyslipidaemia, beyond LDL cholesterol levels.
\end{abstract}

Key words: ezetimibe, cardiovascular risk, atherosclerosis, dyslipidaemia.

Ezetimibe represents the first of a new class of agents, the cholesterol absorption inhibitors, able to reduce low-density lipoprotein (LDL) cholesterol by $15-25 \%$ from baseline in monotherapy and on top of statins and fibrates [1]. The combination with simvastatin represents the most common combined therapy, due to the fact that ezetimibe can add an extra $20 \%$ reduction in LDL cholesterol to that seen with statins alone [2]. Also, ezetimibe is proved to be effective in conditions associated with dyslipidaemia [3-6].

Yet, it seems that ezetimibe produces quantitative rather than qualitative changes in LDL, with small net effects on atherogenic dyslipidaemia. This is supported by findings from the Ezetimibe and Simvastatin in Hypercholesterolemia Enhances Atherosclerosis Regression (ENHANCE) study on atherosclerosis progression, where the addition of ezetimibe to simvastatin in patients with heterozygous familial hypercholesterolaemia did not affect the mean change in carotid intimamedia thickness, although a significant reduction in LDL cholesterol levels was observed [7]. The Simvastatin and Ezetimibe in Aortic Stenosis (SEAS) study [8] has further shown that combination treatment with simvastatin
Corresponding author: Manfredi Rizzo, MD, PhD

Department of Internal Medicine and Medical Specialties University of Palermo Via del Vespro 141-90127 Palermo, Italy Phone/Fax +39 0916552945 E-mail: mrizzo@unipa.it 
Table I. Effects of ezetimibe in monotherapy on LDL size and subclasses in hypercholesterolaemic patients (modified from [9])

\begin{tabular}{|lccccccc|}
\hline Authors & Year & Dose [mg/day] & Duration [weeks] & Patients $(n)$ & Selection criteria & Benefit \\
\hline $\begin{array}{l}\text { Farnier et al. } \\
\text { Tribble et al. }\end{array}$ & 2005 & 10 & 12 & 165 & $\begin{array}{c}\text { mixed hyperlipidaemia } \\
\text { without CHD }\end{array}$ & partial \\
\hline Geiss et al. & 2008 & 10 & 5 & 20 & $\begin{array}{c}\text { severe hyperlipoproteinaemia } \\
\text { and CHD }\end{array}$ & no \\
\hline Kalogirou et al. & 2007 & 10 & 16 & 50 & primary dyslipidaemia & no \\
\hline Ose et al. & 2007 & 10 & 12 & 138 & primary hypercholesterolaemia & no \\
\hline Nakou et al. & 2008 & 10 & 24 & 86 & $\begin{array}{c}\text { overweight and obese subjects } \\
\text { with hypes }\end{array}$ \\
\hline
\end{tabular}

CHD - coronary heart disease

significantly reduced LDL cholesterol levels in patients with aortic stenosis, but did not affect the primary end point of aortic valve and cardiovascular events, although a significant reduction in the risk of ischaemic events was reported.

Since these negative findings were obtained despite a significant reduction in LDL cholesterol levels, we have recently suggested that ezetimibe treats mainly $L D L$ cholesterol and not the underlying dyslipidaemia [9]. In fact, several sources of evidence suggest that the "quality" rather than only the "quantity" of LDL exerts a direct influence on cardiovascular risk: LDL comprise multiple distinct subclasses that differ in size, density, physicochemical composition, metabolic behaviour and atherogenicity $[10,11]$. We have recently shown that small, dense LDL are associated with a greater cardiovascular risk $[12,13]$.

Few studies have so far assessed the effects of ezetimibe on LDL size or their subclass distribution in patients with hypercholesterolaemia, and those in monotherapy are summarized in Table I. Overall, ezetimibe showed a limited role in reducing atherogenic small, dense LDL; yet, since most of these trials included patients at higher cardiovascular risk (due to the concomitant presence of obesity, diabetes and the metabolic syndrome), it cannot be fully excluded that this may have affected the results of these studies.

Therefore, available data so far suggest that treatment with ezetimibe, as monotherapy or in combination with simvastatin, significantly reduces LDL cholesterol concentrations but can be associated with the development of a pro-atherogenic LDL subclass profile. This is directly linked to the observation that end-point studies so far have consistently failed to show that the LDL-lowering effect of ezetimibe directly transfers into a corresponding reduction in cardiovascular events. Further, it has recently been highlighted that ezetimibe and its combination with simvastatin still generate 4 billion dollars per year with no evidence of clinical benefit [14].
Future prospective studies are needed to clarify to what extent ezetimibe is able to reduce atherogenic dyslipidaemia, beyond LDL cholesterol levels. Formal cardiovascular outcome trials are underway and these will provide additional insights into the long-term effects of ezetimibe. For instance, the effect of the combination with statins compared to statin monotherapy on cardiovascular end points is currently being examined by the Improved Reduction of Outcomes: Vytorin Efficacy International Trial (IMPROVE-IT), which aims to recruit a very large cohort of patients with acute coronary syndromes with a follow-up period of at least 2.5 years [15].

\section{References}

1. Mikhailidis DP, Wierzbicki AS, Daskalopoulou SS, et al. The use of ezetimibe in achieving low density lipoprotein lowering goals in clinical practice: position statement of a United Kingdom consensus panel. Curr Med Res Opin 2005; 21: 959-69.

2. Neal RC, Jones PH. Complementary therapy to target LDL cholesterol: the role of the ezetimibe/simvastatin combination. Vasc Health Risk Manag 2006; 2: 31-8.

3. Paraskevas KI, Karatzas G, Pantopoulou A, Iliopoulos DG, Perrea D. Targeting dyslipidemia in the metabolic syndrome: an update. Curr Vasc Pharmacol 2010; 8: 450-63.

4. Angelopoulos J, Krassakopoulos N, Nathanson R, Boukas S, Sampalis JS. Co-administration of ezetimibe and a statin in management of dyslipidemias: a meta-analysis of clinical trials. Arch Med Sci 2009; 5: 347-363.

5. Kalogirou M, Tsimihodimos V, Saougos V, Lagos K, Tselepis AD, Elisaf M. Effect of ezetimibe on lipoprotein subfraction concentrations: the role of atorvastatin pretreatment. Arch Med Sci 2007; 3: 344-350.

6. Grigore L, Norata GD, Catapano AL. Combination therapy in cholesterol reduction: focus on ezetimibe and statins. Vasc Health Risk Manag 2008; 4: 267-78.

7. Kastelein JJ, Akdim F, Stroes ES, et al.; ENHANCE Investigators. Simvastatin with or without ezetimibe in familial hypercholesterolemia. N Engl J Med 2008; 358: 1431-43.

8. Rossebø AB, Pedersen TR, Boman K, et al.; SEAS Investigators. Intensive lipid lowering with simvastatin 
and ezetimibe in aortic stenosis. N Engl J Med 2008; 359: 1343-56.

9. Rizzo M, Rini GB, Spinas GA, Berneis K. The effects of ezetimibe on LDL-cholesterol: quantitative or qualitative changes? Atherosclerosis 2009; 204: 330-3.

10. Rizzo M, Berneis K, Zeljkovic A, Vekic J. Should we routinely measure low-density and high-density lipoprotein subclasses? Clin Lab 2009; 55: 421-9.

11. Gazi IF, Tsimihodimos V, Tselepis AD, Elisaf M Mikhailidis DP. Clinical importance and therapeutic modulation of small dense low-density lipoprotein particles. Expert Opin Biol Ther 2007; 7: 53-72.

12. Berneis K, Rizzo M, Spinas, GA, et al. The predictive role of atherogenic dyslipidemia in subjects with non-coronary atherosclerosis. Clin Chim Acta 2009; 406: 36-40.

13. Rizzo M, Pernice V, Frasheri A, et al. Small, dense lowdensity lipoproteins are predictors of cardio- and cerebrovascular events in subjects with the metabolic syndrome. Clin Endocrinol 2009; 70: 870-5.

14. Mitka M. Cholesterol drug lowers LDL-C levels but again fails to show clinical benefit. JAMA 2010; 303: 211-2.

15. Cannon CP, Giugliano RP, Blazing MA, et al. Rationale and design of IMPROVE-IT (IMProved Reduction of Outcomes: Vytorin Efficacy International Trial): comparison of ezetimibe/simvastatin versus simvastatin monotherapy on cardiovascular outcomes in patients with acute coronary syndromes. Am Heart J 2008; 156: 826-32. 